Liegeois, FJ; Butler, J; Morgan, AT; Clayden, JD; Clark, CA; (2015) Anatomy and lateralization of the human corticobulbar tracts: an fMRI-guided tractography study. Brain Structure and Function 10. 1007/s00429-015-1104-x. (In press). Downloaded from UCL Discovery:

http://discovery.ucl.ac.uk/1471260

\title{
ARTICLE
}

\section{Anatomy and lateralization of the human corticobulbar tracts: an fMRI guided tractography study}

Frédérique J. Liégeois ${ }^{1}$, James Butler ${ }^{1}$, Angela T. Morgan, ${ }^{2-3}$ Jonathan D. Clayden, ${ }^{4}$ Chris A. Clark $^{4}$

1. Cognitive Neuroscience and Neuropsychiatry section, University College London Institute of Child Health, London, United Kingdom

2. Murdoch Children's Research Institute, Melbourne, Australia

3. Department of Pediatrics, University of Melbourne, Australia

4. Developmental Imaging and Biophysics Section, University College London Institute of Child Health, London, United Kingdom

Corresponding author: Frédérique J. Liégeois, Cognitive Neuroscience and Neuropsychiatry section, UCL Institute of Child Health, 30 Guilford Street, London WC1N1EH, United Kingdom, Phone: +44 207905 2728. Fax:+44 207905 2616. E-mail: f.liegeois@ucl.ac.uk.

\section{Abstract}

The left hemisphere lateralization bias for language functions, such as syntactic processing and semantic retrieval, is well known. Although several theories and clinical data indicate a link between speech motor execution and language, the functional and structural brain lateralization for these functions has never been examined concomitantly in the same individuals. Here we used functional MRI during rapid silent syllable repetition (/lalala/, /papapa/ and /pataka/, known as oral diadochokinesis or DDK) to map the cortical representation of the articulators in 17 healthy adults. In these same participants, functional lateralization for language production was assessed using the well established verb generation task. We then used DDK-related fMRI activation clusters to guide tractography of the corticobulbar tract from diffusion-weighted MRI. Functional MRI revealed a wide interindividual variability of hemispheric asymmetry patterns (left and right dominant, as well as bilateral) for DDK in the motor cortex, despite predominantly left hemisphere dominance for language-related activity in Broca's area. Tractography revealed no evidence for structural 
asymmetry (based on fractional anisotropy) within the corticobulbar tract. To our knowledge, this study is the first to reveal that motor brain activation for syllable repetition is unrelated to functional asymmetry for language production in adult humans. In addition, we found no evidence that the human corticobulbar tract is an asymmetric white matter pathway. We suggest that the predominance of dysarthria following left hemisphere infarct is probably a consequence of disrupted feedback or input from left hemisphere language and speech planning regions, rather than structural asymmetry of the corticobulbar tract itself.

Keywords: corticobulbar tract; speech; lateralization; functional MRI; tractography; diffusionweighted MRI.

\section{Introduction}

Speech production is a complicated process, performed by a widely distributed network of brain regions involved in motor planning, syntactic and semantic retrieval, articulation, and auditory feedback processing, among others (see Price, 2010, for a review and Eickhoff et al., 2009, for a network analysis). Evidence from both functional imaging studies in healthy adults (Price 2010; Price 2012; Price et al. 2011) and clinical studies of adults with aphasic disorders post stroke (e.g., Turkeltaub et al. 2011; see Berthier et al. 2011 for a recent review) suggests a leftward asymmetry for linguistic functions such as semantic retrieval and syntactic processing. Although some reports of adults post stroke with deficits in speech motor control, i.e. dysarthria, similarly suggest a left hemisphere functional dominance for articulation (Terao et al. 2007; Urban et al. 2006), we have little direct evidence of functional or structural lateralization for the motor execution aspects of speech. Significant advances in functional MRI and in the tracking of white matter pathways now allow us to reconstruct the corticobulbar tract (CBT) and test the leftward asymmetry hypothesis for speech execution directly.

Diffusion-weighted magnetic resonance imaging (DW-MRI) allows virtual reconstruction of neural pathways by tracking the three-dimensional diffusion of water molecules. This method can be used to examine structural lateralization of white matter tracts in the human brain (Catani et al. 2005; Glasser and Rilling 2008; Filler, 2009), as derived from volumetric or microstructural properties such as fractional anisotropy (FA). DW-MRI tractography has revealed a leftward volumetric asymmetry of the arcuate fasciculus (AF), a major language pathway linking frontal and temporal language regions (Catani et al. 2007; FernandezMiranda et al. 2014; Nucifora et al. 2005; Parker et al. 2005; Thiebaut de Schotten et al. 2011). In addition, preliminary evidence suggests an association between arcuate fasciculus asymmetry (for FA) and functional asymmetry for language during a verb generation task 
(Powell et al. 2006) but this finding has not been replicated in all subsequent studies (e.g., Vernooij et al. 2007). For some cognitive functions, there may therefore be a correlation between functional and structural asymmetry in the human brain.

Recent studies have used DW-MRI techniques to map the course of the adult corticobulbar tract (Akter et al. 2011; Pan et al. 2012; Yim et al. 2013), the direct motor pathways controlling the cranial nerves responsible for articulation. However, none of these studies examined the hemispheric asymmetry of diffusion metrics. Furthermore, anatomical landmarks were used to delineate the seed regions necessary for reconstruction of the CBT, typically the "hand knob" area (Akter et al. 2011). This reliance on anatomical landmarks can be problematic as representations of the articulators have been reported to overlap (see Takai et al. 2010 for a review) and may differ in their precise anatomical locations between individuals. Here we mapped the subcomponents of the CBT in individual subjects by using regions of $\mathrm{fMRI}$ activation during distinct articulatory movements as target regions for our tractography based reconstructions.

Oral diadochokinesis (DDK), a task which involves the rapid repetition of single (e.g. /pa/) or multiple (e.g. /pataka/) syllables, is a highly sensitive task for the differential diagnosis of motor speech impairment (Ackermann et al. 1997; Kent et al. 1987; Ogar et al. 2006; Wit et al. 1993; Ziegler 2002). Its main advantage is that it involves the production of real speech sounds through the use of legal syllables, and excludes the confound of semantic content (i.e. meaning) which could result in left lateralized input to the motor cortex. What is assessed, therefore, is the maximal performance of both motor execution and articulatory planning of syllable sequences. Using a silent version of this task allowed us to focus on articulatory rather than phonatory aspects of syllable repetition. In addition, the silent version avoided auditory input, known to modulate activity in the left primary motor cortex (Watkins et al. 2003), as well as prosody and linguistic rythm. We aimed to examine lateralization of the speech execution system in isolation in order to (i) refine current neuro-anatomical models (ii) improve prognosis for individual with unilateral lesions.

Silent DDK allows us to examine the grey and white matter structures necessary for the execution of speech, namely the primary motor (articulator representation) cortex and corticobulbar tracts. This "final motor pathway" is present in all current computational and anatomical models of speech production (e.g. Bohland et al. 2010; Duffau 2015; Eickhoff et al. 2009; Hickok 2012). As a consequence, its integrity is necessary to (though not sufficient for) speech execution.

In the present study, we used fMRI to identify the regions of the primary motor cortex involved in three DDK tasks. Tractography was then performed from these regions to the 
brainstem to isolate subcomponents of the CBT. In addition, we used a verb generation task to examine language lateralization in the same participants. We hypothesized (i) a leftward functional asymmetry for both language and DDK tasks, and (ii) an association between asymmetry of FA within the CBT and asymmetry for DDK functional activation.

\section{Material and Methods}

\section{Participants}

Eighteen right-handed adults (9 male; mean age $24.63 \pm 4.33$ years; age range 19-35) were recruited via a university website dedicated to the recruitment of research participants. All participants were monolingual native speakers of English, and gave their informed written consent. No participant had a history of speech or language difficulties. The study was approved by the University College London ethics committee (ref 3590/001).

\section{Behavioural assessment}

To determine participants' overall level of intellectual function (IQ), the Matrix Reasoning and Vocabulary subtests from the Wechsler Abbreviated Scale of Intelligence (WASI, Wechsler 1999) were used. An adapted version of the Edinburgh Handedness Inventory (Oldfield

assessed

hand

dominance

(available

at

http://www.brainmapping.org/shared/Edinburgh.php). To assess individual performance on the DDK tasks, the shortest time to perform 12 correct syllable repetitions $(4 \mathrm{x} /$ pataka/ /lalala/ or /papapa/) was measured outside the scanner via audiorecordings (recorded using the Audacity software, http://audacity.sourceforge.net/, at a sampling rate of $44100 \mathrm{~Hz}$ ).

\section{MRI data acquisition}

Structural imaging

MRI scanning was performed using a 1.5 T Avanto System (Siemens, Erlangen, Germany) and a 32-channel head coil. First, structural MRI were collected for localization of fMRI activation, using T1-weighted Fast Low Angle Shot images [3D FLASH: repetition time $(T R)=11 \mathrm{~ms}$; echo time $(T E)=4.94 \mathrm{~ms}, F o V=224 \times 256 \mathrm{~mm}$, matrix size $256 \times 256,1 \mathrm{~mm}^{3}$ isotropic voxels, 176 slices/volume].

Functional imaging

Functional MRI data were then acquired using whole-brain 2D echo-planar imaging sequences $(\mathrm{TR}=7236 \mathrm{~ms}$, TE $=30 \mathrm{~ms}$, FoV $=126 \times 126 \mathrm{~mm}$, matrix size $=64 \times 64,3 \mathrm{~mm}$ isotropic voxels, 125 slices/volume).

Five different tasks were presented in a random order. (1) Verb generation: Participants heard nouns presented via earphones (every 2 seconds) and were asked to covertly 
generate single verbs that are semantically related (e.g. "bread"-"SLICE"). This task is widely used to assess hemispheric language dominance in both healthy and clinical populations (e.g. candidates for epilepsy surgery, Liegeois et al., 2004) and has been validated against invasive procedures (Abou-Khalil 2007) (2) Right hand movement: Participants were instructed to tap all fingers of the right hand sequentially and as quickly as possible. (3-5) Syllable repetition: Participants were instructed to silently repeat syllables as quickly as possible. For the two monosyllabic tasks, participants repeated /lalala/ and /papapa/, predominantly corresponding to movements of the tongue and lips/jaw respectively. A third multisyllabic task required repetition of /pataka/, which involves both movements and coordination of the tongue, lip and jaw.

A block design was used, consisting of 30 seconds ( 6 volumes) of Task, followed by 30 seconds of Rest, for 10 repetitions. During the Rest period, participants were instructed to do nothing and relax. Participants had their eyes open for the duration of the scan, and Task instructions (i.e. "move" or "/lalala/" or "/papapa/" or "/pataka/") were relayed via earphones.

Diffusion-weighted imaging

Lastly, DW-MRI data were collected using a diffusion sensitized EPI sequence $(b=1000 \mathrm{~s}$ $\mathrm{mm}^{2}, 60$ diffusion sensitized directions, $T R=7300 \mathrm{~ms}$, TE $=81 \mathrm{~ms}$, FoV $=192 \times 192 \mathrm{~mm}$, matrix size $=96 \times 96$, slice thickness $=2.5 \mathrm{~mm}$ ) providing $2.5 \mathrm{~mm}$ isotropic voxels.

\section{fMRI data analysis}

Preprocessing

The dataset from one male participant had to be excluded from further analysis as he had not performed the DDK tasks correctly based on his own post scan feedback. Functional MRI data was processed using Statistical Parametric Mapping 8 (SPM8) software (Wellcome Department of Cognitive Neurology, London, UK). Preprocessing steps included spatial realignment to the first dataset, coregistration to the respective T1-weighted images, and normalization onto the default SPM template (Montreal Neurological Institute, from 152 healthy adults). Realignment parameters showed that translation and rotation never exceeded $2 \mathrm{~mm}$ and $2^{\circ}$ respectively. Lastly, the normalised functional images were smoothed with an 8mm FWHM isotropic Gaussian filter.

\section{Activation maps}

For each of the $5 \mathrm{fMRI}$ tasks, activation maps were generated using Task vs. Rest contrasts for each individual. MNI coordinates were converted into Talairach coordinates (Talairach 1988) using WFU PickAtlas (Maldjian et al. 2003), which were used to identify the location of activated clusters. For illustration purposes, group- averaged maps were also created using 
a second level Random effect analysis, with an uncorrected threshold of $p=0.001$ (extent $=20$ voxels).

Lateralization index

Masks for the $\mathrm{LI}$ analysis were generated using the SPM toolbox software Wake Forest University (WFU) PickAtlas (Maldjian et al. 2003). For the verb generation task, a mask covering Broca's area was used, while for the motor tasks a primary motor cortex mask was used.

Hemispheric lateralization was calculated from the individual normalised activation maps using the Lateralization Index (LI) toolbox for SPM8 (Wilke and Lidzba 2007). The LI toolbox uses the following equation to calculate lateralization in activity between the two hemispheres: (Activity Left- Activity Right)/(Activity Left + Right). The resulting LI values range from -1 to 1 . LI values between 1 and 0.2 were categorized as left lateralised, and LIs between -0.2 and -1 were categorized as right lateralised. The remainder were categorized as bilateral. The bootstrap method of analysis was used to calculate an LI (Wilke and Schmithorst 2006), whereby 100 re-samples are taken from each hemisphere at different thresholds.

\section{$D W-M R I$ and tractography analysis}

Preprocessing

DICOM files were converted into Analyze format using the TractoR package (Clayden 2011). The data were then corrected for eddy current-induced distortion using FSL (http://www.fmrib.ox.ac.uk/fsl). Next, TractoR was used to calculate diffusion tensors using standard ordinary least squares fitting, and then the FSL BEDPOSTX algorithm (Behrens et al. 2007) was used to estimate fibre orientations for tractography, allowing up to two fibres per voxel.

Region of interest masks

MarsBar (Brett 2002) was used to extract unilateral primary motor activation regions from the fMRI DDK tasks (significant at $p=0.001$, uncorrected for multiple comparisons), to be used as masks to define the region of interest $(\mathrm{ROI})$ for the tractography. These images were then converted to diffusion space using FMRIB's Linear Image Registration Tool (Jenkinson et al. 2002). A second ROI consisting of a brain stem mask specific to the relevant hemisphere was also created using the WFU Pickatlas (Maldjian et al. 2003). 


\section{Tractography}

Tractography of the whole brain was performed, using all voxels with an FA of at least 0.2 as seed points and retaining only those streamlines that passed through both ROls outlined above. An exclusion mask for the contralateral hemisphere was also used to ensure specificity of tracts to the hemisphere of interest. Voxels through which less than $1 \%$ of the streamlines passed were discarded. TractoR was used to calculate average FA values from all voxels visited by the reconstructed tract, after applying the $1 \%$ visitation threshold.

\section{Statistical analysis}

Numerical data were analysed within SPSS version 20 (SPSS Inc., Chicago). Correlations between Lateralization indices from DDK and verb generation tasks were assessed using Pearson's correlation coefficient $(r)$. A repeated measures analysis of variance (ANOVA) was used to assess differences in LI values between the different fMRI tasks. Differences in DDK speed for the different tasks were also assessed using a repeated measures ANOVA. For DW-MRI data, related samples Wilcoxon signed rank tests were used to assess hemispheric differences. Correlations between structural and functional LIs were assessed using Spearman's correlation coefficient $\left(r_{s}\right)$, as the assumption of normal distribution of the data was violated.

Results

\section{Descriptive measures}

All participants were right-handed (mean handedness score $82.22 \pm 18.09$, range 40 to 100 ) with IQs in the high average range (mean $119.18 \pm 9.24$, range 104 to 133). DDK speed outside the scanner was not statistically different between tasks ( $p>0.05$ in all cases).

The five fMRI tasks generated diverse activation patterns (Figure 1; see supplementary Table 1 for peak coordinates). The /lalala/ and /papapa/ DDK tasks revealed activation within partly overlapping areas corresponding to the classically defined tongue and lips area of the sensorimotor cortex respectively (Grabski et al. 2012). Activation for the multisyllabic DDK task encompassed sensorimotor areas from both simple speech tasks, with additional involvement of the left rolandic operculm and of the putamen bilaterally.

\section{Functional lateralization for language and hand movements}

Motor activation for the right hand task was strongly left lateralized (mean 0.97, see Table 1). Similarly, activation in Broca's area during verb generation was mainly left lateralised (Table 1, Figure 2). 
The DDK tasks yielded a heterogeneous spread of LI values (Table 1, Figure 2), with about a third of participants falling into left, right and bilateral categories. There was no significant correlation between Broca's LI during verb generation and primary motor LI during the DDK tasks ("lalala", $r=0.23, p=0.19$; "papapa", $r=0.34, p=0.09$; "pataka", $r=-0.2, p=0.22$ ).

\section{Structural lateralization of the corticobulbar tract}

Tractography successfully identified motor tracts running from the three seed ROls to the brainstem (see Figure 3 for group results). There was no significant difference ( $p>.08$ in all cases) in FA between the left and right hemispheres in the three CBT subcomponents isolated from the "lalala" task (mean left $=0.368 \pm 0.03$; mean right $=0.372 \pm 0.04$ ), from the "papapa" task (mean left $=0.377 \pm 0.02$; mean right $=0.359 \pm 0.04$ ), or from the "pataka" task (mean left $=0.378 \pm 0.03$; mean right $=0.369 \pm 0.03$ ) seed regions. Mean FA LIs fell between 0.06 and 0.12 (average $\mathrm{LI}=0.03 \pm 0.02$ ) indicating symmetry in all participants.

\section{Relationship between structural and functional lateralization}

No significant correlation was found between the $\mathrm{LI}$ of the mean FA of the corticobulbar tracts and the LI of Broca's area during verb generation ('lalala' track, $r_{s}=-0.54, p=0.07$, 'papapa' track, $r_{s}=-0.41, p=0.14$, 'pataka' track, $\left.r_{s}=0.37, p=0.46\right)$. Similarly, structural CBT and functional DDK LIs did not correlate for any of the three tasks ( $p>0.12$ in al cases).

\section{Discussion}

In healthy adults, we found that motor cortex functional lateralization for rapid syllable repetition is distributed along a left-right continuum, even in the presence of strong left hemisphere dominance for language in individual participants. In addition, our tractography reconstruction revealed symmetry of the CBT as it relates to the DDK task.

\section{Functional hemispheric asymmetry for DDK}

The location of average activation during DDK was consistent with that of previously reported fMRI results for the "mouth" (see Brown et al. 2009, and Fox et al. 2001 for fMRI meta-analyses) and the tongue (e.g., Brown et al. 2009; Brown et al. 2008; Lotze et al. 2000; Takai et al. 2010) sensorimotor regions. It is important to note that although there was no functional asymmetry at the group level in these studies and ours, we observed a wide range of individual functional lateralization patterns, including right and left dominance. One early study had reported left pre-central gyrus activation at the group level during the repetition of /pataka/ (Riecker et al. 2000, in10 right handed participants), which we did not observe. Of note, individual lateralization patterns were not reported in these studies nor was lateralization quantified, therefore comparison with our findings remains limited. 
As previously observed in studies examining repetition of syllables (Lotze et al. 2000; Riecker et al. 2000) even at lower statistical thresholds and at the individual level, the DDK tasks we used did not induce fMRI activation in Broca's area or the anterior insular cortexboth considered classical "speech planning" regions (Dronkers 1996; Hillis et al. 2004). Instead the network of activity was highly restricted to the sensorimotor cortex with extension into the left rolandic operculum for the multisyllabic task. Lesion to the rolandic operculum has been associated with articulatory impairment and buccofacial apraxia (Tonkonogy and Goodglass 1981). More recent neuroimaging studies indicate that this region is commonly activated in tasks that involve articulation (Brown et al. 2009 for a meta-analysis of oral reading and singing). In addition, this region has been shown to be structurally and functionally atypical in people who stutter (Sommer et al. 2002; Watkins et al. 2008). We therefore hypothesize that the detected activity in the left rolandic operculum during complex DDK here is a result of increased planning and programming demand of multisyllabic repetition relative to mono-syllabic repetition.

The involvement of left hemisphere regions outside the primary motor cortex has however been reported by several other groups. One study (Bohland and Guenther 2006) showed bilateral $\mathrm{fMRI}$ activity in the sensorimotor cortex but reported increased left lateralization in the left premotor cortex and prefrontal sulcus with increased sequence complexity-as well as increased striatal involvement (also found here). Another study similarly reported increased left posterior inferior frontal gyrus activation with increased onset syllable complexity (Riecker et al. 2008) when reading bisyllabic pseudowords, whereas reiteration of monosyllables elicited bilateral activation (Riecker et al. 2000). Others have also reported left lateralization in Broca's area and the anterior insular cortex (Riecker et al. 2006; Riecker et al. 2005) during syllable repetition alongside bilateral sensorimotor activation.

Altogether, the available literature is consistent with a lack of left lateralization for syllable repetition within the motor cortex -when examined at the group level. Left hemisphere dominance is mainly seen consistently in other frontal cortical regions involved in planning, such as the rolandic operculum, premotor and inferior frontal cortices. At the individual level, which to our knowledge has not been explored before, we show that lateralization patterns within the motor cortex vary widely. Such variability is in striking contrast to a consistently symmetrical corticobulbar tract (CBT, see below).

\section{Relationship between functional and structural asymmetry}

The lack of relationship between structural (white matter) and functional asymmetry for DDK is consistent with that reported for laryngeal control, where similarly functional but not 
structural asymmetry (as derived using tractography) has been identified (Simonyan et al. 2009). Given that our DDK tasks were executed without voicing (silent articulation) one could argue that the lack of left dominance for DDK induced activation was due to the lack of laryngeal contribution. Indeed, previous reports of left hemisphere dominance for syllable production did involve voicing. This methodological discrepancy raises the possibility that previously reported left hemisphere dominance for syllable repetition is related to voicing or even auditory processing feedback. Here we found no evidence of an association between structural and functional lateralization for silent syllable repetition. We showed that this lack of association is caused by a highly variable speech motor functional asymmetry, in the presence of symmetrical CBTs.

\section{Study limitations}

One might argue that primary motor activity for a silent DDK task is not directly comparable to that for speech articulation. Only a further study comparing motor asymmetry during silent connected speech and during DDK would allow us to answer the question of generalizability of our functional asymmetry findings to speech articulation. Clinical evidence however, suggests that DDK and speech rely on extremely overlapping neuroanatomical substrateswhere impairment on DDK and speech co-occur (Kent and Kent 2000). Several studies have reported a significant correlation between speaking rate and DDK rates in patients with dysarthria (Nishio and Niimi 2006), with DDK rates accounting for $70 \%$ of the variance in severity ratings in ataxic dysarthria (Ziegler and Wessel 1996). As a result, DDK is often used as a clinical tool to identify early signs of neuromuscular dysfunction, with changes in DDK rate more sensitive than speech rate to identify dysarthria in progressive and degenerative diseases (Kent 2000). Evidently, our silent DDK task does not allow us to examine the influence of sensory feedback, linguistic input or prosodic modulation on motor activation. Further research controlling for these influences will shed light on the role of these systems on motor lateralization for speech.

\section{Clinical implications}

In adults, the seminal study by Urban and colleagues (Urban et al. 2006) revealed that infarcts to the left hemisphere result in more frequent and more severe articulation deficits than right hemisphere infarcts. The authors concluded on "an asymmetry of descending cortico-bulbar projections relevant for speech articulation". Our results are not consistent with this interpretation, as we found no evidence of an asymmetrical CBT in our group of adults. In contrast, our findings of highly variable functional lateralization for DDK are in agreement with other reports of acute dysarthria following right hemisphere infarcts (one case/6 in Kim et al. 2003; $10 \%$ of patients in Urban et al. 2006; less than a third in Kumral et 
al. 2007; see Dyukova et al. 2010 for a review). An early study by Urban and colleagues (Urban et al. 1997) for instance had reported on 18 patients with mild to moderate dysarthria, 6 of whom had suffered a right hemisphere infarct. In a later study (Urban et al. 2001), the same group reported that $18.5 \%$ of their 68 consecutive patients with dysarthria had right sided lesions.

If our functional asymmetry findings can be applied to speech motor control, then we predict that right hemisphere infarcts in adults would also result in dysarthria, likely to be observed in the proportion of people who show right hemisphere sensorimotor dominance for DDK. Although in a relatively small cohort, our results indicate that, based on our thresholds for left, right lateralized and bilateral, this is approximately $35 \%$ of the right handed population. Similarly, the symmetrical CBT can explain the fast recovery of dysarthria in most adults with unilateral stroke (Riecker et al. 2002; Urban et al., 2006);

\section{Conclusion}

Our fMRI activation-guided tractography approach allowed us to examine structural white matter and functional lateralization for syllable repetition in the same individuals, and to relate this to language lateralization. Contrary to expectations, there was no relationship between structural and functional asymmetry for these DDK tasks, with a range of left dominant, bilateral and right dominant patterns of functional activation seen. Furthermore, there was no relationship between lateralization of activation induced by verb generation and that from syllable repetition tasks. This study also adds to accumulating evidence that functional asymmetries in the brain are not necessarily associated with structural white matter asymmetries of related pathways. 


\section{Acknowledgement}

We thank our research radiographer, Tina Banks, for scanning and our participants for taking part.

\section{Figure captions}

Fig. 1 Group averaged fMRI activation projected on the cortical surface of a typical rendered brain for the (a) right hand task, (b) verb generation task, (c) 'lalala' DDK task, (d) 'papapa' DDK task, (e) 'pataka' DDK task ( $p<0.001$, uncorrected, Random effect analysis). Left hemisphere is on the right

Fig.2 Distribution of lateralization values across the 17 participants for the five fMRI tasks. Each circle represents one participant. Positive values are left lateralized; negative values right lateralized. Values between -0.2 and +0.2 are categorised as bilateral.

Fig. 3 Group overlay maps, in coronal maximum intensity projection, of the three CBT subsections isolated by tractography between fMRI ROls (see Fig. 1) and the brainstem. Colour gradient indicates number of participants.

Right hemisphere $(R)$ is on the left.
a. /lalala/ task (tongue)
b. /papapa/ task (lips/jaws)
c. /pataka/ task (lips/jaw/tongue) 


\section{References}

Abou-Khalil B (2007) An update on determination of language dominance in screening for epilepsy surgery: the Wada test and newer noninvasive alternatives. Epilepsia 48:442-455 doi:10.1111/j.1528-1167.2007.01012.x

Ackermann H, Konczak J, Hertrich I (1997) The temporal control of repetitive articulatory movements in Parkinson's disease. Brain and language 56:312-319 doi:10.1006/brln.1997.1851

Akter M, Hirai T, Sasao A, Nishimura S, Uetani H, Iwashita K, Yamashita Y (2011) Multitensor tractography of the motor pathway at 3T: a volunteer study. Magnetic resonance in medical sciences : MRMS : an official journal of Japan Society of Magnetic Resonance in Medicine 10:59-63

Behrens TE, Berg HJ, Jbabdi S, Rushworth MF, Woolrich MW (2007) Probabilistic diffusion tractography with multiple fibre orientations: What can we gain? Neurolmage 34:144155 doi:10.1016/j.neuroimage.2006.09.018

Berthier ML et al. (2011) Recovery from post-stroke aphasia: lessons from brain imaging and implications for rehabilitation and biological treatments. Discovery medicine 12:275289

Bohland JW, Guenther FH (2006) An fMRI investigation of syllable sequence production. Neurolmage 32:821-841 doi:10.1016/j.neuroimage.2006.04.173

Bohland JW, Bullock D, Guenther FH (2010) Neural representations and mechanisms for the performance of simple speech sequences Journal of cognitive neuroscience 22:1504-1529 doi:10.1162/jocn.2009.21306

Brett MA, J.-L.; Valabregue, R.; Poline, J.B. (2002) Region of interest analysis using an SPM toolbox. Paper presented at the 8th International Conference on Functional Mapping of the Human Brain, Sendai, Japan,

Brown S, Laird AR, Pfordresher PQ, Thelen SM, Turkeltaub P, Liotti M (2009) The somatotopy of speech: phonation and articulation in the human motor cortex. Brain and cognition 70:31-41 doi:10.1016/j.bandc.2008.12.006

Brown S, Ngan E, Liotti M (2008) A larynx area in the human motor cortex Cereb Cortex 18:837-845 doi:10.1093/cercor/bhm131

Brown S, Laird AR, Pfordresher PQ, Thelen SM, Turkeltaub P, Liotti M (2009) The somatotopy of speech: phonation and articulation in the human motor cortex Brain and cognition 70:31-41 doi:10.1016/j.bandc.2008.12.006

Catani M, Allin MP, Husain M, Pugliese L, Mesulam MM, Murray RM, Jones DK (2007) Symmetries in human brain language pathways correlate with verbal recall. 
Proceedings of the National Academy of Sciences of the United States of America 104:17163-17168 doi:10.1073/pnas.0702116104

Catani M, Jones DK, ffytche DH (2005) Perisylvian language networks of the human brain. Annals of neurology 57:8-16 doi:10.1002/ana.20319

Clayden JDMM, S.; Storkey, A.J.; King, A.D.; Bastin, M.E.; Clark, C.A. (2011) TractoR: Magnetic resonance imaging and tractography with R. Journal of Statistical Software $44: 18$

De Bodt MS, Hernandez-Diaz HM, Van De Heyning PH (2002) Intelligibility as a linear combination of dimensions in dysarthric speech Journal of communication disorders $35: 283-292$

Dronkers NF (1996) A new brain region for coordinating speech articulation. Nature 384:159161 doi:10.1038/384159a0

Duffau $\mathrm{H}$ (2015) Stimulation mapping of white matter tracts to study brain functional connectivity Nature reviews Neurology doi:10.1038/nrneurol.2015.51

Dyukova GM, Glozman ZM, Titova EY, Kriushev ES, Gamaleya AA (2010) Speech disorders in right-hemisphere stroke Neuroscience and behavioral physiology. 40:593-602 doi:10.1007/s11055-010-9301-9

Eickhoff SB, Heim S, Zilles K, Amunts K (2009) A systems perspective on the effective connectivity of overt speech production. Philosophical transactions Series A, Mathematical, physical, and engineering sciences 367:2399-2421 doi:10.1098/rsta.2008.0287

Fernandez-Miranda JC, Wang Y, Pathak S, Stefaneau L, Verstynen T, Yeh FC (2014) Asymmetry, connectivity, and segmentation of the arcuate fascicle in the human brain. Brain structure \& function doi:10.1007/s00429-014-0751-7

Fox PT, Huang A, Parsons LM, Xiong JH, Zamarippa F, Rainey L, Lancaster JL (2001) Location-probability profiles for the mouth region of human primary motor-sensory cortex: model and validation. Neurolmage 13:196-209 doi:10.1006/nimg.2000.0659

Glasser MF, Rilling JK (2008) DTI tractography of the human brain's language pathways. Cereb Cortex 18:2471-2482 doi:10.1093/cercor/bhn011

Grabski K et al. (2012) Functional MRI assessment of orofacial articulators: neural correlates of lip, jaw, larynx, and tongue movements. Human brain mapping 33:2306-2321 doi:10.1002/hbm.21363

Hickok G (2012) Computational neuroanatomy of speech production Nature reviews Neuroscience 13:135-145 doi:10.1038/nrn3158

Hillis AE, Work M, Barker PB, Jacobs MA, Breese EL, Maurer K (2004) Re-examining the brain regions crucial for orchestrating speech articulation. Brain : a journal of neurology 127:1479-1487 doi:10.1093/brain/awh172 
Jenkinson M, Bannister P, Brady M, Smith S (2002) Improved optimization for the robust and accurate linear registration and motion correction of brain images. Neurolmage 17:825-841

Kent RD, Kent JF, Rosenbek JC (1987) Maximum performance tests of speech production. The Journal of speech and hearing disorders 52:367-387

Kent RD, Kent JF (2000) Task-based profiles of the dysarthrias Folia Phoniatr Logop 52:4853 doi:21512

Kent RD (2000) Research on speech motor control and its disorders: a review and prospective Journal of communication disorders 33:391-427.

Kim JS, Kwon SU, Lee TG (2003) Pure dysarthria due to small cortical stroke Neurology 60:1178-1180

Kumral E, Celebisoy M, Celebisoy N, Canbaz DH, Calli C (2007) Dysarthria due to supratentorial and infratentorial ischemic stroke: a diffusion-weighted imaging study. Cerebrovasc Dis 23:331-338 doi:10.1159/000099131

Liegeois F, Connelly A, Cross JH, Boyd SG, Gadian DG, Vargha-Khadem F, Baldeweg T (2004) Language reorganization in children with early-onset lesions of the left hemisphere: an fMRI study. Brain : a journal of neurology 127:1229-1236 doi:10.1093/brain/awh159

Lotze M, Seggewies G, Erb M, Grodd W, Birbaumer N (2000) The representation of articulation in the primary sensorimotor cortex. Neuroreport 11:2985-2989

Maldjian JA, Laurienti PJ, Kraft RA, Burdette JH (2003) An automated method for neuroanatomic and cytoarchitectonic atlas-based interrogation of fMRI data sets. Neurolmage 19:1233-1239

Nishio M, Niimi S (2006) Comparison of speaking rate, articulation rate and alternating motion rate in dysarthric speakers Folia Phoniatr Logop 58:114-131 doi:10.1159/000089612

Nucifora PG, Verma R, Melhem ER, Gur RE, Gur RC (2005) Leftward asymmetry in relative fiber density of the arcuate fasciculus. Neuroreport 16:791-794

Ogar J, Willock S, Baldo J, Wilkins D, Ludy C, Dronkers N (2006) Clinical and anatomical correlates of apraxia of speech. Brain and language 97:343-350 doi:10.1016/j.bandl.2006.01.008

Oldfield RC (1971) The assessment and analysis of handedness: The Edinburgh inventory Neuropsychologia 9:97-113

Pan C, Peck KK, Young RJ, Holodny AI (2012) Somatotopic organization of motor pathways in the internal capsule: a probabilistic diffusion tractography study. AJNR American journal of neuroradiology 33:1274-1280 doi:10.3174/ajnr.A2952 
Parker GJ, Luzzi S, Alexander DC, Wheeler-Kingshott CA, Ciccarelli O, Lambon Ralph MA (2005) Lateralization of ventral and dorsal auditory-language pathways in the human brain. Neurolmage 24:656-666 doi:10.1016/j.neuroimage.2004.08.047

Powell HW et al. (2006) Hemispheric asymmetries in language-related pathways: a combined functional MRI and tractography study. Neurolmage 32:388-399 doi:10.1016/j.neuroimage.2006.03.011

Price CJ (2010) The anatomy of language: a review of $100 \mathrm{fMRI}$ studies published in 2009. Annals of the New York Academy of Sciences 1191:62-88 doi:10.1111/j.17496632.2010.05444.x

Price CJ (2012) A review and synthesis of the first 20 years of PET and fMRI studies of heard speech, spoken language and reading. Neurolmage 62:816-847 doi:10.1016/j.neuroimage.2012.04.062

Price CJ, Crinion JT, Macsweeney M (2011) A Generative Model of Speech Production in Broca's and Wernicke's Areas. Frontiers in psychology 2:237 doi:10.3389/fpsyg.2011.00237

Riecker A, Ackermann H, Wildgruber D, Meyer J, Dogil G, Haider H, Grodd W (2000) Articulatory/phonetic sequencing at the level of the anterior perisylvian cortex: a functional magnetic resonance imaging (fMRI) study. Brain and language 75:259-276 doi:10.1006/brln.2000.2356

Riecker A, Brendel B, Ziegler W, Erb M, Ackermann H (2008) The influence of syllable onset complexity and syllable frequency on speech motor control. Brain and language 107:102-113 doi:10.1016/j.bandl.2008.01.008

Riecker A, Kassubek J, Groschel K, Grodd W, Ackermann H (2006) The cerebral control of speech tempo: opposite relationship between speaking rate and BOLD signal changes at striatal and cerebellar structures. Neurolmage 29:46-53 doi:10.1016/j.neuroimage.2005.03.046

Riecker A, Mathiak K, Wildgruber D, Erb M, Hertrich I, Grodd W, Ackermann H (2005) fMRI reveals two distinct cerebral networks subserving speech motor control. Neurology 64:700-706 doi:10.1212/01.WNL.0000152156.90779.89

Riecker A, Wildgruber D, Grodd W, Ackermann H (2002) Reorganization of speech production at the motor cortex and cerebellum following capsular infarction: a followup functional magnetic resonance imaging study. Neurocase 8:417-423 doi:10.1076/neur.8.5.417.16181

Simonyan K, Ostuni J, Ludlow CL, Horwitz B (2009) Functional but not structural networks of the human laryngeal motor cortex show left hemispheric lateralization during syllable but not breathing production The Journal of neuroscience : the official journal of the Society for Neuroscience 29:14912-14923 doi:10.1523/JNEUROSCI.4897-09.2009 
Sommer M, Koch MA, Paulus W, Weiller C, Buchel C (2002) Disconnection of speechrelevant brain areas in persistent developmental stuttering Lancet 360:380-383 doi:10.1016/s0140-6736(02)09610-1

Takai O, Brown S, Liotti M (2010) Representation of the speech effectors in the human motor cortex: somatotopy or overlap? Brain and language 113:39-44 doi:10.1016/j.bandl.2010.01.008

Talairach JT, P. (1988) Co-planar Stereotaxic Atlas of the Human Brain. Thieme Medical, New York, USA

Terao $Y$ et al. (2007) Primary face motor area as the motor representation of articulation. Journal of neurology 254:442-447 doi:10.1007/s00415-006-0385-7

Thiebaut de Schotten M et al. (2011) Atlasing location, asymmetry and inter-subject variability of white matter tracts in the human brain with MR diffusion tractography. Neurolmage 54:49-59 doi:10.1016/j.neuroimage.2010.07.055

Tonkonogy J, Goodglass H (1981) Language function, foot of the third frontal gyrus, and rolandic operculum Archives of neurology 38:486-490

Turkeltaub PE, Messing S, Norise C, Hamilton RH (2011) Are networks for residual language function and recovery consistent across aphasic patients? Neurology 76:1726-1734 doi:10.1212/WNL.0b013e31821a44c1

Urban PP, Hopf HC, Fleischer S, Zorowka PG, Muller-Forell W (1997) Impaired corticobulbar tract function in dysarthria due to hemispheric stroke. Functional testing using transcranial magnetic stimulation. Brain : a journal of neurology 120 ( Pt 6):10771084

Urban PP, Rolke R, Wicht S, Keilmann A, Stoeter P, Hopf HC, Dieterich M (2006) Lefthemispheric dominance for articulation: a prospective study on acute ischaemic dysarthria at different localizations. Brain : a journal of neurology 129:767-777 doi:10.1093/brain/awh708

Urban PP et al. (2001) Dysarthria in acute ischemic stroke: lesion topography, clinicoradiologic correlation, and etiology. Neurology 56:1021-1027

Vernooij MW, Smits M, Wielopolski PA, Houston GC, Krestin GP, van der Lugt A (2007) Fiber density asymmetry of the arcuate fasciculus in relation to functional hemispheric language lateralization in both right- and left-handed healthy subjects: a combined fMRI and DTI study. Neurolmage 35:1064-1076 doi:10.1016/j.neuroimage.2006.12.041

Wechsler D (1999) Wechsler Abbreviated Scale of Intelligence ${ }^{\mathrm{TM}}$ (WASI $^{\mathrm{TM}}$ ) Pearson Assessment, London, UK 
Watkins KE, Smith SM, Davis S, Howell P (2008) Structural and functional abnormalities of the motor system in developmental stuttering Brain : a journal of neurology 131:50-59 doi:10.1093/brain/awm241

Watkins KE, Strafella AP, Paus T (2003) Seeing and hearing speech excites the motor system involved in speech production Neuropsychologia 41:989-994

Wilke M, Lidzba K (2007) LI-tool: a new toolbox to assess lateralization in functional MRdata Journal of neuroscience methods. 163:128-136 doi:10.1016/j.jneumeth.2007.01.026

Wilke M, Schmithorst VJ (2006) A combined bootstrap/histogram analysis approach for computing a lateralization index from neuroimaging data. Neurolmage 33:522-530 doi:10.1016/j.neuroimage.2006.07.010

Wit J, Maassen B, Gabreels FJ, Thoonen G (1993) Maximum performance tests in children with developmental spastic dysarthria. Journal of speech and hearing research 36:452-459

Yim SH et al. (2013) Distribution of the corticobulbar tract in the internal capsule. Journal of the neurological sciences 334:63-68 doi:10.1016/j.jns.2013.07.015

Ziegler W (2002) Task-related factors in oral motor control: speech and oral diadochokinesis in dysarthria and apraxia of speech. Brain and language 80:556-575 doi:10.1006/brln.2001.2614

Ziegler W, Wessel K (1996) Speech timing in ataxic disorders: sentence production and rapid repetitive articulation Neurology 47:208-214 


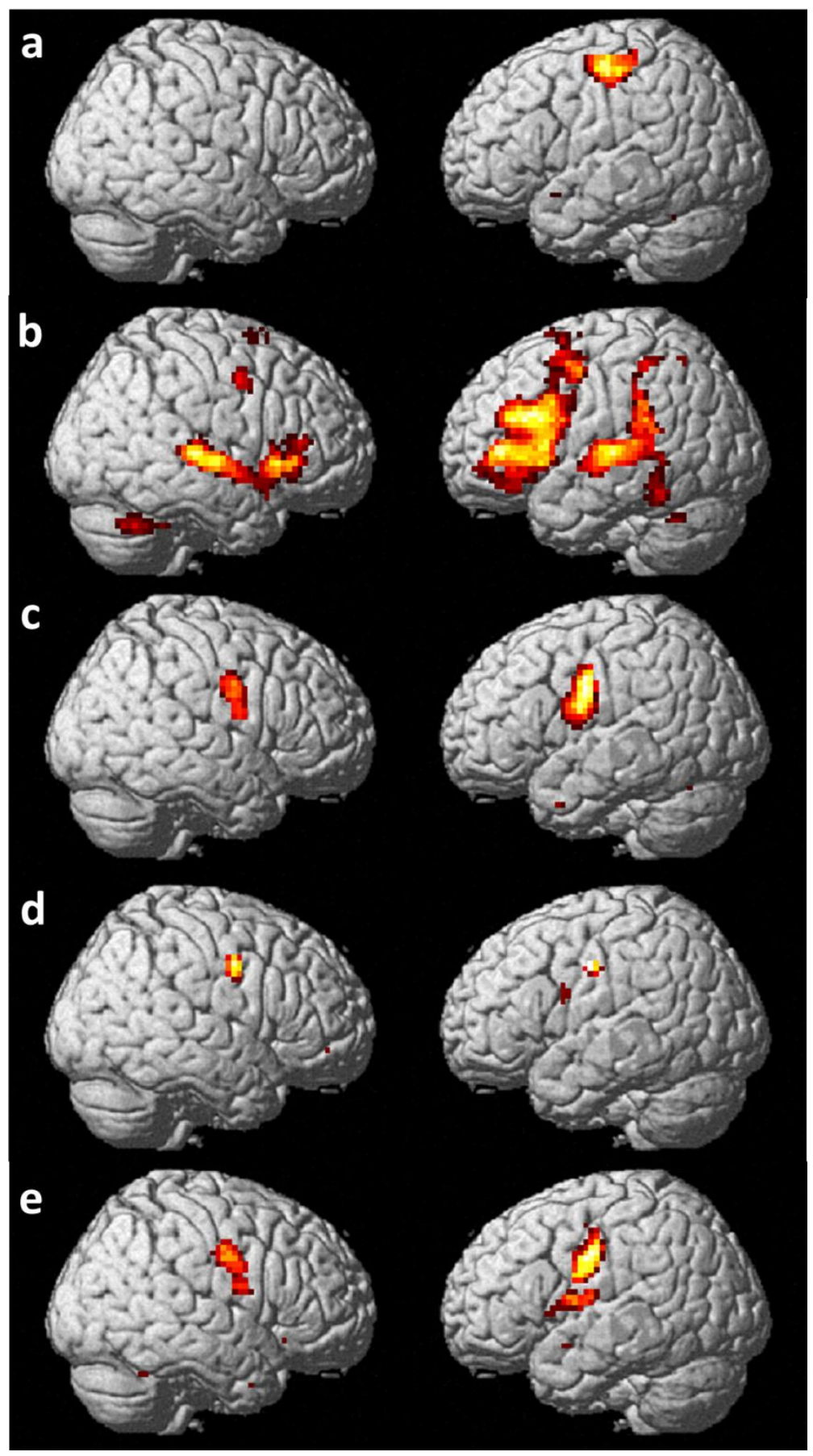

Fig.1. Group averaged fMRI activation projected on the cortical surface of a typical rendered brain for the (a) right hand task, (b) verb generation task, (c) "lalala" DDK task, (d) "papapa" DDK task, (e) "pataka" DDK task ( $p<0.001$, uncorrected, Random effect analysis). Left hemisphere is on the right. 


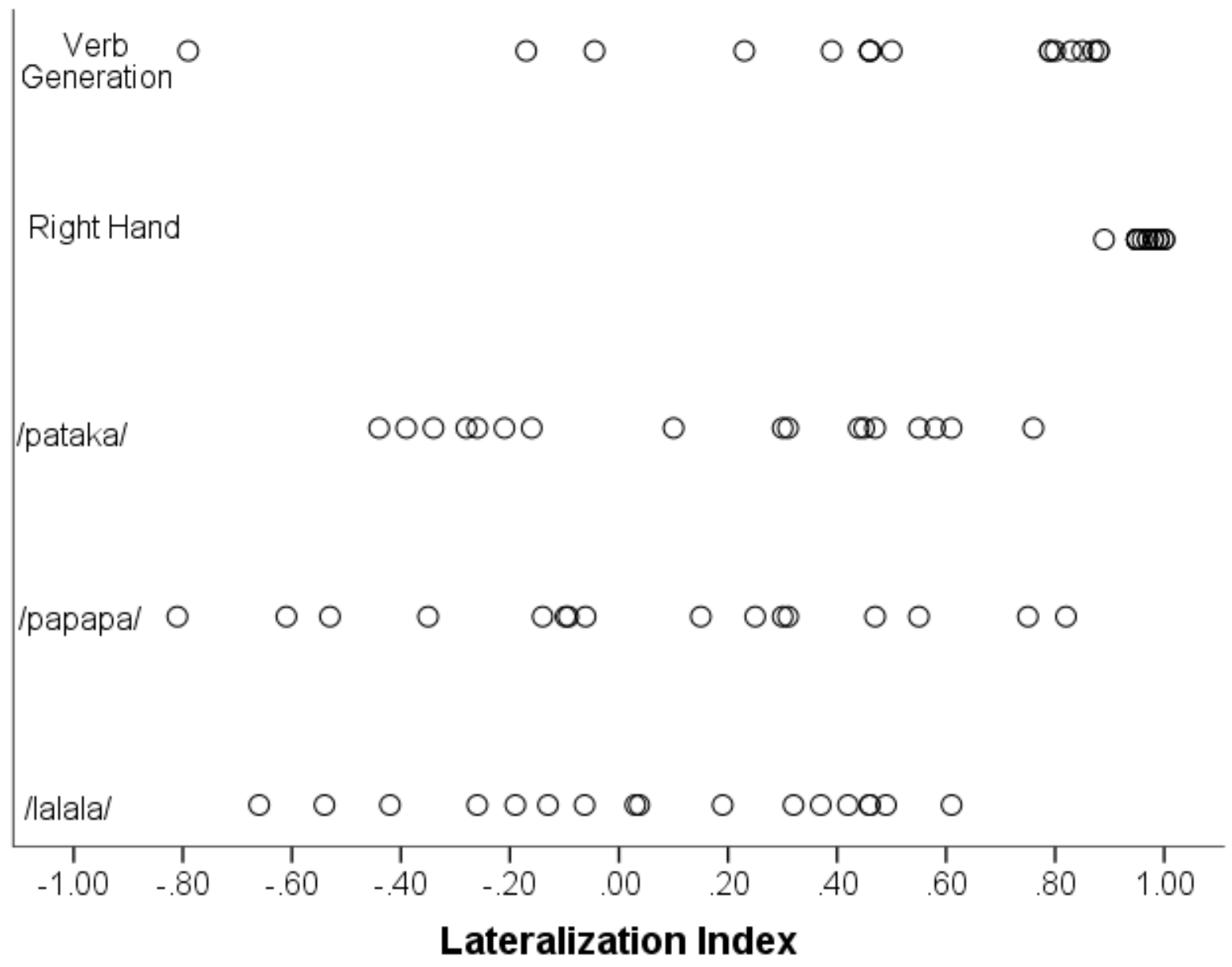

Figure 2. Distribution of lateralization values across the 17 participants for the five fMRI tasks. Each circle represents one participant. Positive values are left lateralized; negative values right lateralized. Values between -0.2 and +0.2 are categorised as bilateral. 


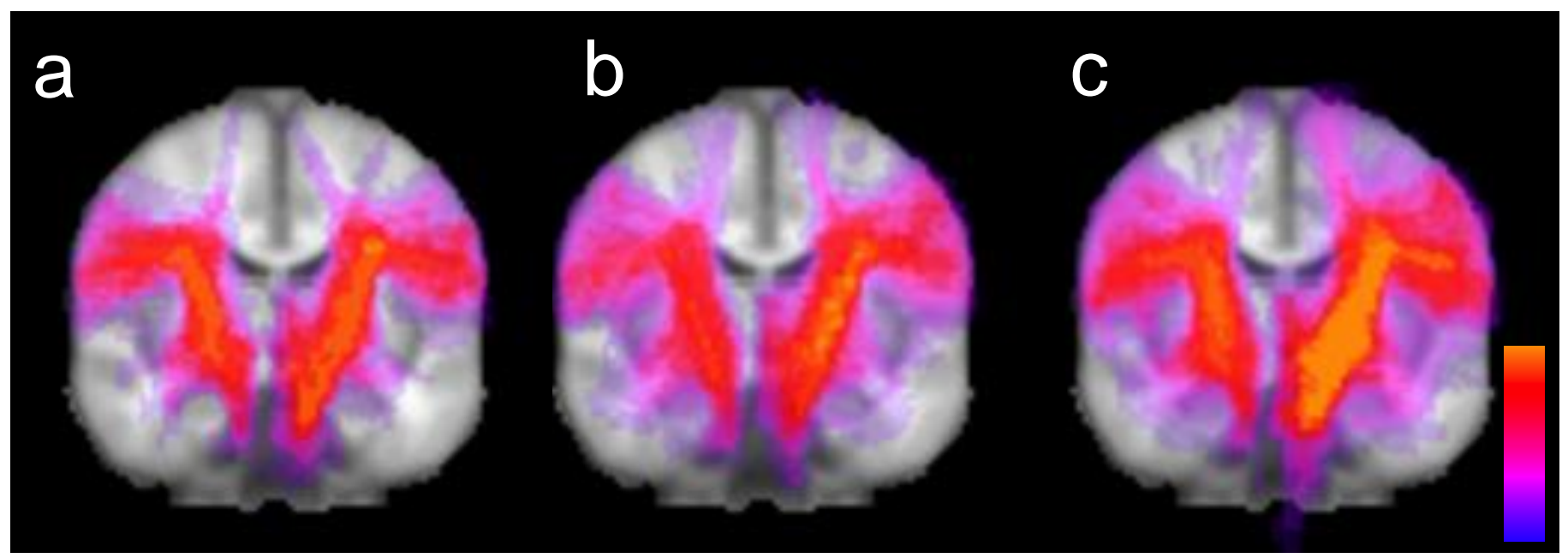

Figure 3. Group overlay maps, in coronal maximum intensity projection, of the three CBT subsections isolated by tractography between fMRI ROls (see Fig. 1) and the brainstem. Colour gradient indicates number of participants.

Right hemisphere $(R)$ is on the left.
a. /lalala/ task (tongue)
b. /papapa/ task (lips/jaws)
c. /pataka/ task (lips/jaw/tongue) 
Supplementary Table A1. fMRI results: Task vs. baseline contrasts for the five tasks used.

\begin{tabular}{|c|c|c|c|c|c|}
\hline Task & $\begin{array}{l}\text { Anatomical } \\
\text { location }\end{array}$ & $\begin{array}{l}\text { Peak } \\
\text { coordinate }\end{array}$ & $\begin{array}{l}\text { Cluster } \\
\text { size }\end{array}$ & $\begin{array}{l}\text { Significance } \\
\text { level (cluster, } \\
\text { FDR } \\
\text { corrected) }\end{array}$ & $\mathrm{Z}$ value at peak \\
\hline \multirow[t]{2}{*}{ Right hand } & $\begin{array}{l}\text { Left precentral } \\
\text { gyrus }\end{array}$ & $-36-1955$ & 302 & $<0.001$ & $6.56^{*}$ \\
\hline & $\begin{array}{l}\text { Right cerebellum } \\
\text { Lob. V }\end{array}$ & $15-49-17$ & 267 & $<0.001$ & $5.62^{*}$ \\
\hline \multirow[t]{7}{*}{$\begin{array}{l}\text { Verb } \\
\text { Generation }\end{array}$} & $\begin{array}{l}\text { Left inferior frontal } \\
\text { gyrus, dorsal }\end{array}$ & -331128 & 2271 & $<0.001$ & $5.13^{*}$ \\
\hline & $\begin{array}{l}\text { Right post. superior } \\
\text { temporal gyrus }\end{array}$ & $69-227$ & 285 & $<0.001$ & $4.89^{*}$ \\
\hline & $\begin{array}{l}\text { Left post. superior } \\
\text { temporal gyrus }\end{array}$ & $-45-3710$ & 737 & $<0.001$ & $4.76^{*}$ \\
\hline & Right putamen & $2711-5$ & 514 & $<0.001$ & $4.73^{*}$ \\
\hline & $\begin{array}{l}\text { Right cerebellum } \\
\text { Lob. VI }\end{array}$ & $24-64-35$ & 334 & $<0.001$ & $4.66^{*}$ \\
\hline & $\begin{array}{l}\text { Left cerebellum } \\
\text { Lob. VI }\end{array}$ & $-30-52-38$ & 41 & 0.05 & 4.22 \\
\hline & & $57-143$ & 33 & 0.07 & 3.81 \\
\hline \multirow[t]{4}{*}{ "Lalala" } & $\begin{array}{l}\text { Left postcentral } \\
\text { gyrus }\end{array}$ & $-60-422$ & 259 & $<0.001$ & 4.84 \\
\hline & $\begin{array}{l}\text { Right postcentral } \\
\text { gyrus }\end{array}$ & $54-131$ & 115 & 0.008 & 4.39 \\
\hline & $\begin{array}{l}\text { Right cerebellum, } \\
\text { Lob. VI }\end{array}$ & $15-61-26$ & 71 & 0.031 & 4.13 \\
\hline & $\begin{array}{l}\text { Left cerebellum, } \\
\text { Lob. VI }\end{array}$ & $-24-61-23$ & 59 & 0.04 & 3.78 \\
\hline \multirow[t]{3}{*}{ "Papapa" } & $\begin{array}{l}\text { Right precentral } \\
\text { gyrus }\end{array}$ & $48-440$ & 43 & 0.20 & 3.73 \\
\hline & $\begin{array}{l}\text { Left postcentral } \\
\text { gyrus }\end{array}$ & -421037 & 33 & 0.21 & 3.57 \\
\hline & $\begin{array}{l}\text { Right cerebellum } \\
\text { Lob. VI }\end{array}$ & $15-61-17$ & 44 & 0.20 & 3.39 \\
\hline
\end{tabular}




\begin{tabular}{|c|c|c|c|c|c|}
\hline "Pataka" & Left putamen & $-248-14$ & 49 & 0.15 & 4.90 \\
\hline & Right cerebellum & $15-61-20$ & 208 & 0.01 & $4.73^{*}$ \\
\hline & Lob. VI & & & & \\
\hline & Left $\quad$ Cerebellum & $-9-58-23$ & 104 & 0.04 & 4.18 \\
\hline & Lob. VI & & & & \\
\hline & Right putamen & $33-111$ & 25 & 0.34 & 3.98 \\
\hline & $\begin{array}{l}\text { Left precentral } \\
\text { gyrus }\end{array}$ & $-51-728$ & 176 & 0.01 & $3.91^{*}$ \\
\hline & $\begin{array}{l}\text { Left Rolandic } \\
\text { operculum }\end{array}$ & $-63-1013$ & 56 & 0.14 & 3.85 \\
\hline & $\begin{array}{l}\text { Right precentral } \\
\text { gyrus }\end{array}$ & $45-1037$ & 110 & 0.04 & 3.83 \\
\hline
\end{tabular}

$\mathrm{L}$, left hemisphere; $\mathrm{R}$, right hemisphere; post, posterior. extent threshold $=20$ voxels. All coordinates indicate peak voxels significant at $p=0.001$, uncorrected for multiple comparisons. FDR correction is provided for information. *significant at $p<0.05$ with FWE correction at cluster level. Lob, lobule. 